\title{
Active Transport of Nanomaterials Using Motor Proteins
}

\section{Final report for DOE-BES grant DE-FG02-05ER46193}

$(02 / 05-07 / 05)$

\author{
Submitted to: \\ Dr. Timothy Fitzsimmons \\ The Division of Materials Sciences \\ Office of Basic Energy Scienc \\ U.S. Department of Energy
}

\section{Prepared by: Henry Hess, Department of Bioengineering, University of Washington September 2005}

PRINCIPAL INVESTIGATORS: Prof. Henry Hess

\section{SUMMARY}

During the six months of funding we have focused first on the completion of the research begun at the University of Washington in the previous funding cycle. Specifically, we developed a method to polymerize oriented networks of microtubules on lithographically patterned surfaces (M.S. thesis Robert Doot). The properties of active transport have been studied detail, yielding insights into the dispersion mechanisms (Nitta et al.). The assembly of multifunctional structures with a microtubule core has been investigated (Ramachandran et al.).

Isaac Luria (B.S. in physics, U. of Florida 2005) worked on the directed assembly of nanoscale, non-equilibrium structures as a summer intern. He is now a graduate student in my group at the University of Florida. Individual experiments performed for this research project were combined with results from the DARPA Biomolecular Motors program to yield higher impact publications (Nitta and Hess, Ramachandran et al.).

List of accomplishments:

\section{Peer-reviewed journals:}

T. Nitta and H. Hess: "Dispersion in Active Transport by Kinesin-Powered Molecular Shuttles", Nano Letters, 5, 1337-1342 (2005) - partial support from DARPA Biomolecular Motors Program

S. Ramachandran, K.-H. Ernst, G. D. Bachand, V. Vogel, H. Hess*: "Selective Loading of Kinesin-Powered Molecular Shuttles with Protein Cargo and its Application to Biosensing", submitted to Small (2005) - partial support from DARPA Biomolecular Motors Program 
R. Doot, H. Hess, V. Vogel: "Engineering Two-Dimensional Networks of Oriented Microtubules for Directed Cargo Transport" - to be submitted to Nano Letters

\section{Invited Lectures:}

H. Hess, et al.: "Biomolecular Motors: Engines for Nanotechnology", Seminar of the Department of Bioengineering, California Institute of Technology, Pasadena, CA (2005)

H. Hess: "Exploiting the Force-Generating Properties of Microtubule Motors in Nanoscience", Experts Workshop on the Physics of Biological Force Generation, Institute for Complex Adaptive Matter, Snowmass, CO (2005

H. Hess: "Accelerating unbinding with biomolecular motors: Implications for selfassembly", 2005 ACS Annual Meeting, Symposium "Nanoscale Science and Catalysis in Biomolecular Catalysis", Washington, DC (2005)

\section{Supported Students (1 M.S. graduated), Postdocs, and Faculty:}

Henry Hess (Research Assistant Professor - 50\% support)

Robert Doot (graduate Student) 